\title{
Analysis of gallbladder polypoid lesion size as an indication of the risk of gallbladder cancer
}

\author{
Ji Eun Sung, Chang Woo Nam, Yang Won Nah, and Byung Sung Kim
}

\begin{abstract}
Department of Surgery, Ulsan University Hospital and University of Ulsan College of Medicine, Ulsan, Korea
\end{abstract}
\begin{abstract}
Backgrounds/Aims: Recent advances in ultrasonography have contributed to the early detection of gallbladder cancer. We attempted to predict the progression of the disease by comparing the sizes of polypoid lesions, and we suggest that the size of the lesion would be a useful guideline to determine an appropriate primary surgical approach for polypoid lesions of the gallbladder. Methods: We have retrospectively analyzed 253 patients that, during the operation period from January 2009 to December 2011, had had ultrasonographically detected gallbladder polypoid lesions, and who underwent cholecystectomy at Ulsan university hospital. We have analyzed the demographic data of the patients, the preoperative size of polypoid lesions, and pathologic findings. Results: Of a total of 253 patients, 235 patients had benign lesions, and 18 patients had malignant lesions. Among the malignant polyp patients, 11 had pT1 cancer, 6 had pT2 cancer, and 1 had pT3 cancer. The average size of polypoid lesions was $9.1 \pm 3.1 \mathrm{~mm}$ and that of malignant lesions was $28.2 \pm 16.4 \mathrm{~mm}$. The receiver operating characteristic (ROC) curve of the benign and malignant groups shows that $14.5 \mathrm{~mm}$ is the optimal point of prediction of the malignancy. Of a total of 18 patients of GB cancer, 11 had pT1 and the average size of their polypoid lesions was $20.5 \pm 5.8 \mathrm{~mm} 7$ had pT2 with a size of $39.1 \pm 20.7 \mathrm{~mm}$. ROC curve analysis of the pT1 and pT2 groups shows that $27 \mathrm{~mm}$ would be the optimal point to predict T2 and above cancer. Conclusions: In the case of an early cancer, curative treatment can be achieved through a simple and minimally invasive laparoscopic cholecystectomy. We attempted to predict early cancer occurrence among polypoid lesions of the gallbladder using the simplest standard, size. Although there are some limitations, size can be a simple and easy way to evaluate polypoid lesions of the gallbladder. (Korean J Hepatobiliary Pancreat Surg 2014;18:9-13)
\end{abstract}

Key Words: Gallbladder; Polypoid lesion; Gallbladder cancer; Size; Stage

\section{INTRODUCTION}

Recent advances in ultrasonography contributed to the early detection of gallbladder (GB) cancer. Eighty percent of GB cancers are characteristically detected as polypoid lesions, and differential diagnosis is only carried out after pathologic examination. ${ }^{1}$ The treatment of a polypoid lesion of the gallbladder (PLG) has been carried out in accordance with the "Gallbladder polyp practice recommendation" issued by the Korean Association of HepatoBiliary-Pancreatic Surgery (KAHBPS). According to the recommendation, simple cholecystectomy is an adequate treatment for Tis and T1a lesions because there is no difference in survival rate with radical resection in early gallbladder cancer. On the other hand, GB cancer with T2 and above should be treated with radical resection. ${ }^{2}$ Radiologic findings have some limitations in estimating GB cancer and depth of invasion through imaging findings. GB cancer is suspected preoperatively in only $30 \%$ of patients whereas the other $70 \%$ are discovered incidentally after following a simple cholecystectomy for other diseases such as GB stones and GB adenomyomatosis. ${ }^{3,4}$ It is difficult to determine the appropriate surgical approach for PLG preoperatively, especially when neoplastic polypoid lesions are suspected. In this study, we attempted to predict the progression of the disease by comparing the size of polypoid lesions, and we suggest that the size of the lesion would be a useful standard to determine an appropriate primary surgical approach for a PLG.

\section{METHODS}

We obtained data on 253 patients with PLGs that had had a preoperative radiologic examination of the gall-

Received: October 4, 2013; Revised: October 15, 2013; Accepted: November 1, 2013

Corresponding author: Chang Woo Nam

Department of Surgery, Ulsan University Hospital, 290-3, Jeonha-dong, Dong-gu, Ulsan 682-714, Korea

Tel: +82-52-250-7109, Fax: +82-52-250-7350, E-mail: drcwnam@hotmail.com 
bladder and subsequently underwent cholecystectomy between January 2009 and December 2011 at our institution. We analyzed the sex, age, preoperative polypoid lesion size during radiologic examination, surgical method, tissue pathologic findings, $\mathrm{T}$ stages of 253 patients. The Mann-Whitney $U$ test was used to evaluate the correlation between size, malignancy, and $\mathrm{T}$ stages. Receiver operating characteristic (ROC) curve analysis was also performed for correlation analysis.

\section{RESULTS}

\section{Demographic profiles of patients}

During the period from January 2009 to December 2011, 253 patients with PLGs were subjected to a cholecystectomy. Of the 253 patients, 120 (47.4\%) were male and $133(52.6 \%)$ were female, with an average age of 51.6 years (range: 21-87) across both genders.

\section{Surgical procedures}

Of the 253 patients, 239 (94.5\%) underwent a laparoscopic cholecystectomy, and 3 underwent open cholecystectomy $3(1.2 \%)$. Extended cholecystectomy was carried out in 11 patients $(4.4 \%)$.

\section{Preoperative radiological examination}

Preoperative ultrasonography was performed in most of the patients $(90.1 \%)$. In half of the patients, computed tomography and magnetic resonance imaging was performed.

Table 1. Pathologic profiles

\begin{tabular}{lc}
\hline \multicolumn{1}{c}{ Pathologic diagnoses } & No. of patients $(\%)$ \\
\hline Adenocarcinoma & $18(7.1)$ \\
pT1a & 8 \\
pT1b & 3 \\
pT2 & 6 \\
pT3 & 1 \\
Cholesterol polyp & $148(58.5)$ \\
Adenomyomatous polyp & $2(0.8)$ \\
Hyperplastic polyp & $8(3.2)$ \\
Other polyp & $1(0.4)$ \\
Tubular adenoma & $16(6.3)$ \\
Adenomyosis & $3(1.2)$ \\
Cholecystitis & $35(13.8)$ \\
Others & $22(8.7)$ \\
\hline
\end{tabular}

\section{Pathologic profiles}

Of the total 253 patients, benign lesions were identified in 235 patients and malignant lesions were detected in 18 patients. Histologically, the most of common benign gallbladder polyps were cholesterol polyps with a count of 148 cases. 13 were non-cholesterol polyps; 16 were adenoma; 35 were cholecystitis; and 22 showed other benign lesions. Among malignant polyp patients, 11 were pT1 cancer; 6 were pT2 cancer; and 1 was pT3 cancer (Table 1).

\section{Size correlation between benign and malignant lesions}

Of the total 253 patients with PLGs, benign lesions were present in 235 patients, and the average size of the GB polyps was $9.1 \pm 3.1 \mathrm{~mm}$. Malignancy was found in 18 patients and the average polyp size was $28.2 \pm 16.4 \mathrm{~mm}$. There is a statically significant difference in average polyp sizes of the benign and malignant groups $(p<0.001)$. A ROC curve shows $14.5 \mathrm{~mm}$ is the optimal point to predict the malignancy (Fig. 1).

\section{Size comparison between pT1 and pT2 and above gallbladder cancers}

Of the 18 patients with GB cancer, 11 had T1 with an average polyp size of $20.5 \pm 5.8 \mathrm{~mm} ; 7$ patients were T2 and abovewith a size of $39.1 \pm 20.7 \mathrm{~mm}$. There is statically a significant difference in average polyp sizes of the pT1 and pT2 and above groups $(p=0.026)$. A ROC curve shows $27 \mathrm{~mm}$ and less is the optimal range to predict $\mathrm{T} 1$ cancer (Fig. 2).

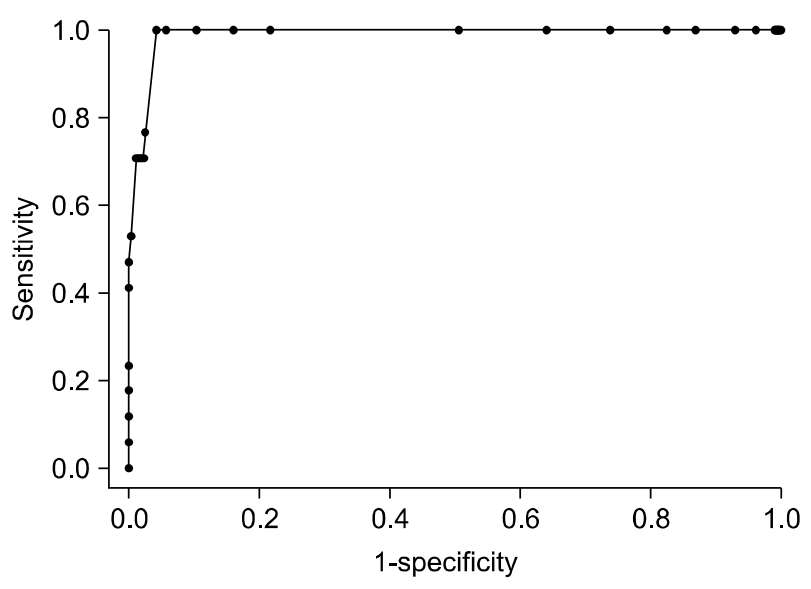

Fig. 1. ROC curve of the correlation between malignant and benign lesions based on data in Table $2.14 .5 \mathrm{~mm}$ is the optimal point to predict malignancy. 
Table 2. Cut-off value to predict malignancy comparing sensitivity and specificity

\begin{tabular}{ccc}
\hline \multicolumn{2}{c}{ Malignancy vs. Benign polyps } & \\
\hline Size cut-off $(\mathrm{mm})$ & Sensitivity & Specificity \\
\hline 0 & 1 & 0 \\
1.5 & 1 & 0.004 \\
2.5 & 1 & 0.009 \\
3.5 & 1 & 0.039 \\
4.5 & 1 & 0.070 \\
5.5 & 1 & 0.131 \\
6.5 & 1 & 0.175 \\
7.5 & 1 & 0.262 \\
8.5 & 1 & 0.358 \\
9.5 & 1 & 0.493 \\
10.5 & 1 & 0.782 \\
11.5 & 1 & 0.838 \\
12.5 & 1 & 0.895 \\
13.5 & 1 & 0.943 \\
14.5 & 1 & 0.956 \\
15.5 & 0.765 & 0.974 \\
16.5 & 0.706 & 0.978 \\
17.5 & 0.706 & 0.983 \\
19 & 0.706 & 0.987 \\
21 & 0.529 & 0.996 \\
23 & 0.471 & 1 \\
27 & 0.412 & 1 \\
32.5 & 0.235 & 1 \\
40 & 0.176 & 1 \\
47.5 & 0.118 & 1 \\
63.5 & 0.059 & 1 \\
78 & 0 & \\
\hline
\end{tabular}

\section{DISCUSSION}

The prevalence of PLGs in healthy subjects varies from $0.004 \%$ to $13.8 \%{ }^{5}$ In Korea, PLG prevalence was $6.1 \%{ }^{6}$ PLGs were first defined pathologically by Christense and Ishak. ${ }^{7}$ Generally, these lesions were categorized into benign and malignant groups. Benign lesions consist of neoplastic polyps (adenoma, hemangioma, lipoma), non-neoplastic polyps (cholesterol polyps, inflammatory polyps, hyperplastic polyps). On other hand, malignant lesions include adenocarcinoma, Squamous cell carcinoma, etc. ${ }^{7,8}$

Adenomas are benign growths in the wall of the GB and are diagnosed in $0.5 \%$ of all cholecystectomy cases. They are usually single lesion and pedunculated. ${ }^{9}$ Most malignant polyps are adenocarcinoma. There are some studies that demonstrate the presence of adenomatous areas in carcinomas as well as the presence of malignant focus in gallbladder adenomas. Kozuka suggested the adenoma-carcinoma carcinogenic sequence. ${ }^{10,11}$
Table 3. Cut-off value to predict $\mathrm{T} 1$ cancer comparing sensitivity and specificity

\begin{tabular}{ccc}
\hline \multicolumn{2}{c}{ Stage pT1 vs. pT2 and higher } & \\
\hline Size cut-off $(\mathrm{mm})$ & Sensitivity & Specificity \\
\hline 14 & 1 & 0 \\
15.5 & 0.857 & 0.300 \\
18 & 0.857 & 0.400 \\
21 & 0.857 & 0.700 \\
23 & 0.714 & 0.700 \\
27 & 0.714 & 0.800 \\
32.5 & 0.571 & 1 \\
40 & 0.429 & 1 \\
47.5 & 0.286 & 1 \\
63.5 & 0.143 & 1 \\
78 & 0 & 1 \\
\hline
\end{tabular}

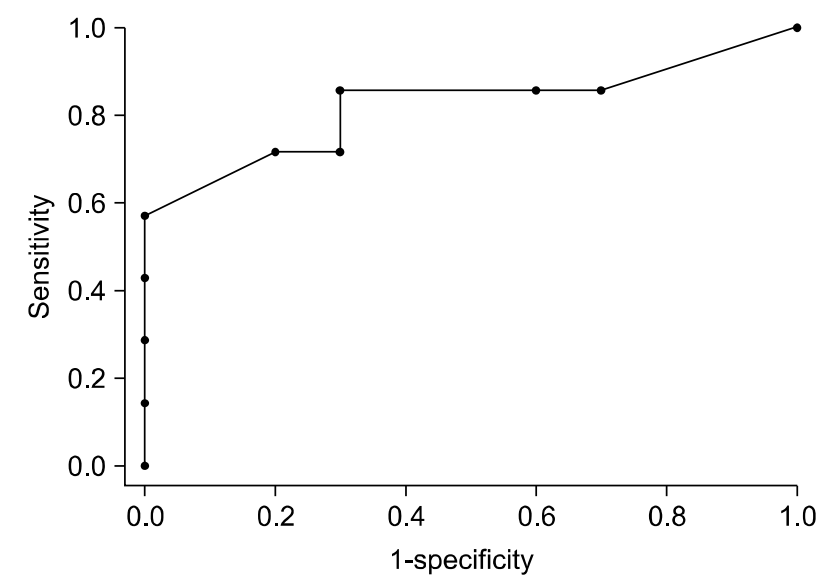

Fig. 2. ROC curve of the correlation between malignant and benign lesions based on data in Table $3.27 \mathrm{~mm}$ would be the optimal point to predict $\mathrm{T} 2$ cancer and higher.

GB cancer is an aggressive malignancy and carries an extremely poor prognosis. The only chance of cure comes from early detection and curative surgery. ${ }^{6}$ There are known risk factors that increase the likelihood of malignancy in a polypoid lesion, and these are size, number, morphologic type and growth rate of polyp, advancing age, and presence of gallstones. ${ }^{12,13}$ Larger poyp size increases the likelihood of malignancy. ${ }^{14,15}$ Terzi et al. reported the histopathologic characteristics of polypoid lesions in 100 patients who had cholecystectomy. Of the 74 patients with benign PLGs, only 11 (15\%) had polyps larger than $10 \mathrm{~mm}$. In contrast, $23(88 \%)$ of the 26 malignant polyps were larger than $10 \mathrm{~mm} .{ }^{16}$ A study in Korea reported that polyps larger than $5 \mathrm{~mm}$ had potential for malignancy and all polyps larger than $15 \mathrm{~mm}$ were malignant. ${ }^{17}$ In these above-mentioned studies, larger pol- 
yp size suggests higher likelihood of malignancy. Generally, a patient who has a PLG larger than $10 \mathrm{~mm}$ would be recommended to undergo cholecystectomy. ${ }^{14,16}$

In patients with PLG larger than $10 \mathrm{~mm}$, there is a dilemma in determining the primary surgical approach. Some patients received simple laparoscopic cholecystectomy, but other patients underwent radical cholecystectomy. According to KAHBP gGuidelines, simple and minimally invasive laparoscopic cholecystectomy is likely to provide an acceptable surgical outcome compared to that of radical surgery in patients who have early GB cancer and hardly suspected GB cancer. However, the depth of invasion can only be confirmed through pathologic examination. Frozen-section biopsy analysis can be helpful during operation, but there are errors in predicting the depth of the lesion in some cases. The larger size of polyp increases the likelihood of malignancy as well as increases the likelihood of advance. We focused on those points and studied how to predict early GB cancer preoperatively using a simple standard, such as polyp size.

In this study, there is a significant difference in the average sizes of malignant $(28.2 \pm 16.4 \mathrm{~mm})$ and benign lesions $(9.1 \pm 3.1 \mathrm{~mm})$. The ROC curve shows $14.5 \mathrm{~mm}$ is the optimal point of predicting the malignancy. We identified there is statically significant difference in average sizes between $\mathrm{T} 1(20.5 \pm 5.8 \mathrm{~mm})$ and $\mathrm{T} 2$ and higher $(39.1 \pm 20.7 \mathrm{~mm}) \mathrm{GB}$ cancers. We also presented that 27 $\mathrm{mm}$ would be the optimal point to predict above T2 cancer through ROC-curve. In patient with a PLG size larger than $14.5 \mathrm{~mm}$ but less than d less $27 \mathrm{~mm}$, although the lesion is suspected as malignancy, the stage is considered as T1 cancer. Laparoscopic cholecystectomy can be performed as an appropriate primary surgical approach reasonably.

We attempted to figure out whether the simplest standard, size of polypoid lesions, can be an obvious criterion in predicting malignancy or invasiveness. This criterion using size is very simple, but cannot be a definite and only way to determine malignancy and invasiveness. Other factors also can affect the risk of malignancy. That might be limitation of this study. Lack of a higher number of patients involved in the study can be another limitation. Furthermore, a large multicenter study will be required to create safe and definite criteria to predict malignancy and invasiveness of PLGs.
Once a malignancy is diagnosed after laparoscopic cholecystectomy, potentially curative therapy should be considered. ${ }^{2}$ The therapeutic surgical treatment of gallbladder cancer according to stage is supported by most surgeons. ${ }^{18-20}$ Most surgeons agree that simple cholecystectomy is an adequate treatment for pTis and pT1a lesions, and that tumors stage pT2 and above should be treated by an additional radical operation. However, there is controversy regarding the management of $\mathrm{T} 1 \mathrm{~b}$ disease. ${ }^{21,22}$ In the present study, only 3 patients had $\mathrm{T} 1 \mathrm{~b}$ tumors and radical surgery was performed in only one case. All T2 and $\mathrm{T} 2$ and above tumor patients underwent radical surgery as a primary or second procedure following simple cholecystectomy.

In summary, the majority of PLGs are benign, and only a small portion thereof develops GB cancer. In the case of an early cancer, curative treatment can be achieved through simple and minimally invasive laparoscopic cholecystectomy. We attempted to predict early cancer within PLGs based on the simplest standard, size. We suggest $14.5 \mathrm{~mm}$ is the optimal point to predict malignancy of PLGs, and $27 \mathrm{~mm}$ is the optimal point to predict $\mathrm{T} 2$ cancer and above. Although there are some limitations, size can be a simple and easy way to evaluate PLGs.

\section{REFERENCES}

1. Choi SB, Han HJ, Kim CY, et al. Incidental gallbladder cancer diagnosed following laparoscopic cholecystectomy. World J Surg 2009;33:2657-2663.

2. Zhang WJ, Xu GF, Zou XP, et al. Incidental gallbladder carcinoma diagnosed during or after laparoscopic cholecystectomy. World J Surg 2009;33:2651-2656.

3. Varshney S, Butturini G, Gupta R. Incidental carcinoma of the gallbladder. Eur J Surg Oncol 2002;28:4-10.

4. Goetze TO, Paolucci V. Adequate extent in radical re-resection of incidental gallbladder carcinoma: analysis of the German Registry. Surg Endosc 2010;24:2156-2164.

5. Cho YK, Park CY, Shin JH. A surgical consideration on gallbladder polyp-a proposal of therapeutic guideline for gallbladder polyp-. Korean J Gastroenterol 2002;39:415-419.

6. Park JK, Yoon YB, Kim YT, et al. Management strategies for gallbladder polyps: is it possible to predict malignant gallbladder polyps? Gut Liver 2008;2:88-94.

7. Christensen AH, Ishak KG. Benign tumors and pseudotumors of the gallbladder. Report of 180 cases. Arch Pathol 1970;90:423432.

8. Yeh CN, Jan YY, Chao TC, et al. Laparoscopic cholecystectomy for polypoid lesions of the gallbladder: a clinicopathologic study. Surg Laparosc Endosc Percutan Tech 2001;11:176-181.

9. Bang S. Natural course and treatment strategy of gallbladder polyp. Korean J Gastroenterol 2009;53:336-340. 
10. Roa I, de Aretxabala X, Araya JC, et al. Preneoplastic lesions in gallbladder cancer. J Surg Oncol 2006;93:615-623.

11. Kozuka S, Tsubone N, Yasui A, et al. Relation of adenoma to carcinoma in the gallbladder. Cancer 1982;50:2226-2234.

12. Myers RP, Shaffer EA, Beck PL. Gallbladder polyps: epidemiology, natural history and management. Can J Gastroenterol 2002; 16:187-194.

13. Shin SR, Lee JK, Lee KH, et al. Can the growth rate of a gallbladder polyp predict a neoplastic polyp? J Clin Gastroenterol 2009;43:865-868.

14. Lee KF, Wong J, Li JC, et al. Polypoid lesions of the gallbladder. Am J Surg 2004;188:186-190.

15. Corwin MT, Siewert B, Sheiman RG, et al. Incidentally detected gallbladder polyps: is follow-up necessary?--Long-term clinical and US analysis of 346 patients. Radiology 2011;258:277-282.

16. Terzi C, Sökmen S, Seçkin S, et al. Polypoid lesions of the gallbladder: report of 100 cases with special reference to operative indications. Surgery 2000;127:622-627.
17. Kim MS, Sim MS. Operative criteria for polypoid lesions of the gallbladder. J Korean Surg Soc 1999;57:107-113.

18. de Groen PC, Gores GJ, LaRusso NF, et al. Biliary tract cancers. N Engl J Med 1999;341:1368-1378.

19. Whalen GF, Bird I, Tanski W, et al. Laparoscopic cholecystectomy does not demonstrably decrease survival of patients with serendipitously treated gallbladder cancer. J Am Coll Surg 2001; 192:189-195.

20. Muratore A, Polastri R, Capussotti L. Radical surgery for gallbladder cancer: current options. Eur J Surg Oncol 2000;26:438443.

21. Wakai T, Shirai Y, Yokoyama N, et al. Early gallbladder carcinoma does not warrant radical resection. Br J Surg 2001;88:675678.

22. Shukla PJ, Barreto G, Kakade A, et al. Revision surgery for incidental gallbladder cancer: factors influencing operability and further evidence for T1b tumours. HPB (Oxford) 2008;10:43-47. 